\title{
1300 nm Fiber Laser System for THG and 2PEF Bio-Imaging
}

\author{
Carsten Cleff ${ }^{1}$, Fernanda Ramos-Gomes ${ }^{2,3}$, Thorsten Bergmann ${ }^{4}$, Luigi Bonacina $^{5}$, Ulrich Weikert ${ }^{6}$, Miso $^{2}$ \\ Mitkovski ${ }^{7}$, Martin Schuette ${ }^{4}$, Frauke Alves ${ }^{2,3}$, and Michael Mei ${ }^{1}$ \\ [1] Menlo Systems GmbH, Am Klopferspitz 19a, 82152 Martinsried, Germany \\ [2] Dept. Molecular Biology of Neuronal Signals, Max-Planck-Institute of Experimental Medicine, Hermann-Rein-Str. 3, D37075 Göttingen \\ [3] Department of Hematology and Oncology, University Medical Center Göttingen, 37075, Germany \\ [4] LaVision BioTec GmbH, Astastr. 14, 33617 Bielefeld, Germany \\ [5] GAP-Biophotonics, Université de Genève, 22 chemin de Pinchat, CH-1211 Genève, Switzerland \\ [6] Department of Cellular Neuroscience, Max-Planck-Institute of Experimental Medicine, Hermann-Rein-Str. 3, 37075 Göttingen, Germany \\ [7] Light Microscopy Facility, Max-Planck-Institute of Experimental Medicine, Hermann-Rein-Str. 3, 37075 Göttingen, Germany \\ c.cleff@menlosystems.com
}

\begin{abstract}
We present a $1300 \mathrm{~nm}$ fiber laser source for bio-imaging applications. We demonstrate nonlinear imaging using nanocrystals as optical markers for THG microscopy and efficient 2photon-excitation of fluorophores at $1300 \mathrm{~nm}$ in biological samples.

OCIS codes: (140.3510) Lasers, fibers (140.7090) Ultrafast lasers; (190.4370) Nonlinear Optics, fibers; (180.4315) Nonlinear Microscopy
\end{abstract}

\section{Introduction}

Nonlinear microscopy techniques have become an import tool in bio-imaging applications, offering advantages over traditional linear microscopy techniques and new possibilities for sample investigation [1]. Nonlinear microscopy offers an intrinsic 3-dimensional high resolution imaging capability and allows for the use of longer excitation wavelengths which enables deeper penetration into biological samples while causing less photo damage. Using excitation wavelengths between 700 and $1080 \mathrm{~nm}$ nonlinear microscopy can be performed using common Titanium:Sapphire laser systems, however, an extension to higher wavelengths requires expensive laser systems such as optical parametric oscillators (OPO) or optical parametric amplifiers (OPA). As a result, despite the advantage for bio-imaging, wavelength above $1080 \mathrm{~nm}$ are rarely used.

Here, we present a fiber laser system designed for bio-imaging at $1300 \mathrm{~nm}$ to provide cost-efficient access to the benefits of deeper sample penetration and reduced sample damage at longer excitation wavelength. We demonstrate bio-imaging of biological samples using different fluorophores and markers at $1300 \mathrm{~nm}$ excitation wavelength and low power levels.

\section{Experimental Setup}

For nonlinear bio-imaging the $1300 \mathrm{~nm}$ femtosecond pulses were free-space coupled into a commercial nonlinear microscope (LaVision BioTec,TRiMScope II) which allows for rapid multimodal imaging. The laser beam was focused onto the sample using a high NA microscope objective. For image acquisition the laser beam was scanned over the sample using a set of galvanometer scanning mirrors. The generated signal was collected in epidirection by the focusing microscope objective and directed onto photomultipliers after spectral filtering. Image acquisition times were typically around $1 \mathrm{~s}$ for images of 512x512 pixels. At a second input port of the nonlinear microscope a femtosecond Titanium:Sapphire laser (tunable from $700-1050 \mathrm{~nm}$ ) was coupled in for two-photonfluorescence (2PEF) and second harmonic generation (SHG) microscopy providing multimodal imaging.

\section{Results}

The laser system is based on an ytterbium fiber laser (Orange, Menlo Systems) and a fiber-based frequency conversion unit. The fiber laser emits femtosecond pulses with an average power of up to $1 \mathrm{~W}$ at $1040 \mathrm{~nm}$ and at a repetition rate of $100 \mathrm{MHz}$. About a quarter of the power at $1040 \mathrm{~nm}$ is used for nonlinear frequency conversion generating $62 \mathrm{~mW}$ of average power at $1300 \mathrm{~nm}$, of which a maximum power of $15 \mathrm{~mW}$ is available at the sample plane. The remaining power at $1040 \mathrm{~nm}$ is available at an optional second output port and can be used simultaneously to the $1300 \mathrm{~nm}$ emission. A wavelength tunability from 1200-1350 nm can be achieved by changing the pulse duration or power of the pump light at $1040 \mathrm{~nm}$.

Figure 1(a) shows a projection of a 2PEF image z-stack acquired from a $15 \mu$ m-thick p20 mouse brain cortex, where microtubule associated protein 2 (MAP2) was stained with Alexa Fluor 647 and excited at $1300 \mathrm{~nm}$ with the 
fiber laser system. The MAP2 signal in this case is used to investigate neuronal dendritic processes at different stages of mouse brain development. Besides fluorophores we also used BFO nanoparticles as optical markers for bio-imaging as they show low cytotoxicity [2] and generate strong non-resonant SHG and THG signals over a wide range of excitation wavelengths $[3,4]$. They can be ingested by phagocytes, working as a tracking molecule to trace the phagocytes with nonlinear microscopy. Figure 1(b) shows images of BFO particles on a cover slide acquired with third harmonic generation (THG) microscopy at $1300 \mathrm{~nm}$ (top, green) and SHG microscopy at $840 \mathrm{~nm}$ (bottom, red). THG and SHG images were taken sequentially and detected by the same photomultiplier equipped with a bandpass filter centered at $420 \mathrm{~nm}$. Despite the longer excitation wavelength, THG imaging with the fiber laser system at $1300 \mathrm{~nm}$ provided better spatial resolution than SHG imaging with the Titanium:Sapphire laser at $840 \mathrm{~nm}$ resulting in a much sharper image of the BFO nanoparticle (fig. 1 (b) top, green).

Figure 2 shows images of H8N8 mouse mammary tumor cells which were incubated overnight in a medium containing BFO particles of $100-150 \mathrm{~nm}$ size. In fig. 2(a) a THG image at $1300 \mathrm{~nm}$ excitation of the sample is shown only displaying the BFO particles. 2PEF images of the sample at $820 \mathrm{~nm}$ excitation are shown in fig. 2(b) and (c) showing the cell membrane (stained with T-Antigen in green) and nuclear (stained with DRAQ5 in red), respectively. Figure 2 (d) shows the merger of images (a)-(c), showing that the tumor cells are able to phagocyte the BFO particles successfully.

In conclusion, we have shown a cost-efficient femtosecond fiber laser source at $1300 \mathrm{~nm}$ presenting an alternative to currently available complex and expensive laser sources for $1300 \mathrm{~nm}$ emission. We demonstrated the suitability of our laser system for bio-imaging by acquiring high contrast images of biological samples at high speed and low power levels. In THG imaging we found strongly improved images using the fiber laser source at $1300 \mathrm{~nm}$ compared to SHG imaging with a Titanium:Sapphire laser.
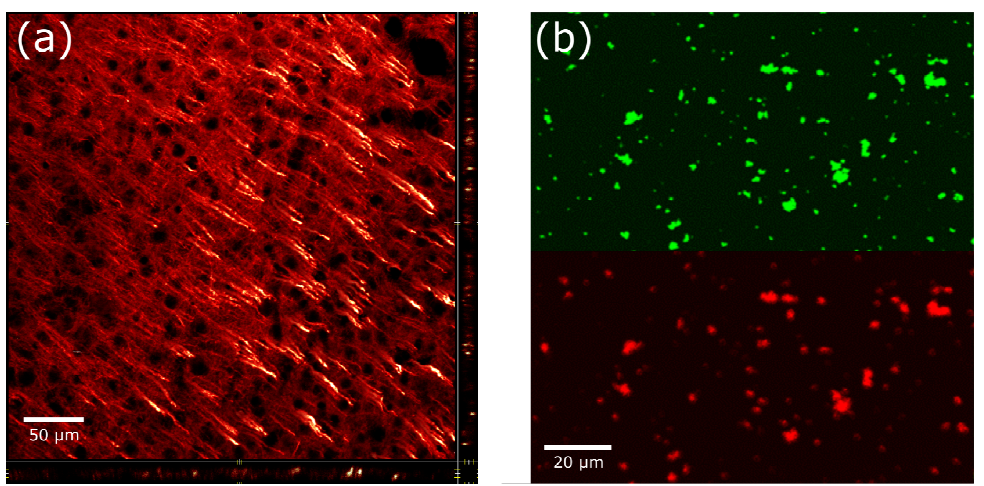

Figure 1: (a) 2PEF image of a $15 \mu \mathrm{m}$ thick mouse brain slice excited at $1300 \mathrm{~nm}$. (b) top: THG image of BFO nanoparticles excited by the fiber laser system at $1300 \mathrm{~nm}$ and (bottom) SHG image of the same sample excited with a Titanium:Sapphire laser at $840 \mathrm{~nm}$
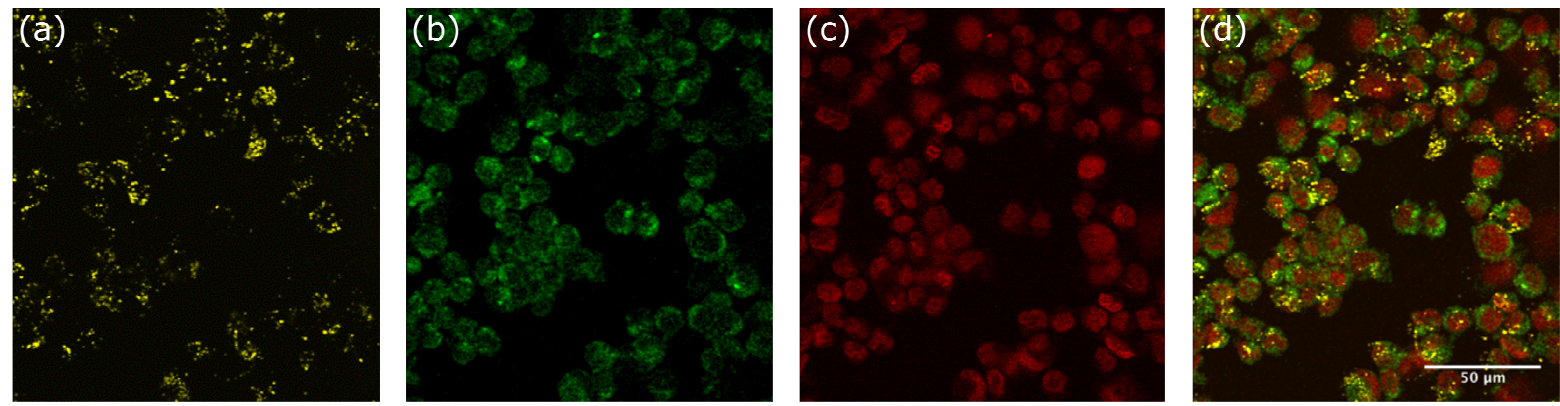

Figure 2: H8N8 mouse mammary tumor cells which phagocyte BFO particles. (a) THG image of BFO particles excited at 1300 nm. (b) 2PEF image of T-Antigen excited at $820 \mathrm{~nm}$ used for staining the cell membrane. (c) 2PEF image of DRAQ5 excited at $820 \mathrm{~nm}$ used for staining the nuclear. (d) Merged image of (a), (b), and (c).

\section{References}

[1] D. Debarre et al., "Imaging lipid bodies in cells and tissues using third-harmonic generation microscopy", Nature Methods 3, 47 (2006).

[2] D. Staedler et al., "Cellular uptake and biocompatibility of bismuth ferrite harmonic advanced nanoparticles", Nanomedicine: NBM 11, 815824 (2015).

[3] A. Rogov et al., "Simultaneous Multiharmonic Imaging of Nanoparticles in Tissues for Increased Selectivity", ACS Photonics 2, 1416-1422 (2015).

[4] A. Rogov et al., "Harmonic nanoparticles: noncentrosymmetric metal oxides for nonlinear optics", J. Opt. 17, 033001 (2015). 